\title{
Not During the Past Month
}

National Cancer Institute

\section{Source}

National Cancer Institute. Not During the Past Month. NCI Thesaurus. Code C121608.

An indication that something has not occurred during the past month. 